\title{
(2) Shape and Color Features for Object Recognition Search
}

\section{This technique can be used in Internet image searching, intelligent video, and security and} surveillance applications.

\section{NASA's Jet Propulsion Laboratory, Pasadena, California}

A bio-inspired shape feature of an object of interest emulates the integration of the saccadic eye movement and horizontal layer in vertebrate retina for object recognition search where a single object can be used one at a time. The optimal computational model for shapeextraction-based principal component analysis (PCA) was also developed to reduce processing time and enable the real-time adaptive system capability. A color feature of the object is employed as color segmentation to empower the shape feature recognition to solve the object recognition in the heterogeneous environment where a single technique - shape or color - may expose its difficulties. To enable the effective system, an adaptive architecture and autonomous mechanism were developed to recognize and adapt the shape and color feature of the moving object.

The bio-inspired object recognition based on bio-inspired shape and color can be effective to recognize a person of interest in the heterogeneous environment where the single technique exposed its difficulties to perform effective recognition. Moreover, this work also demonstrates the mechanism and architecture of the autonomous adaptive system to enable the realistic system for the practical use in the future.

This work was done by Tuan A. Duong and Vu A. Duong of Caltech, and Allen R. Stubberud of UCI for NASA's Jet Propulsion

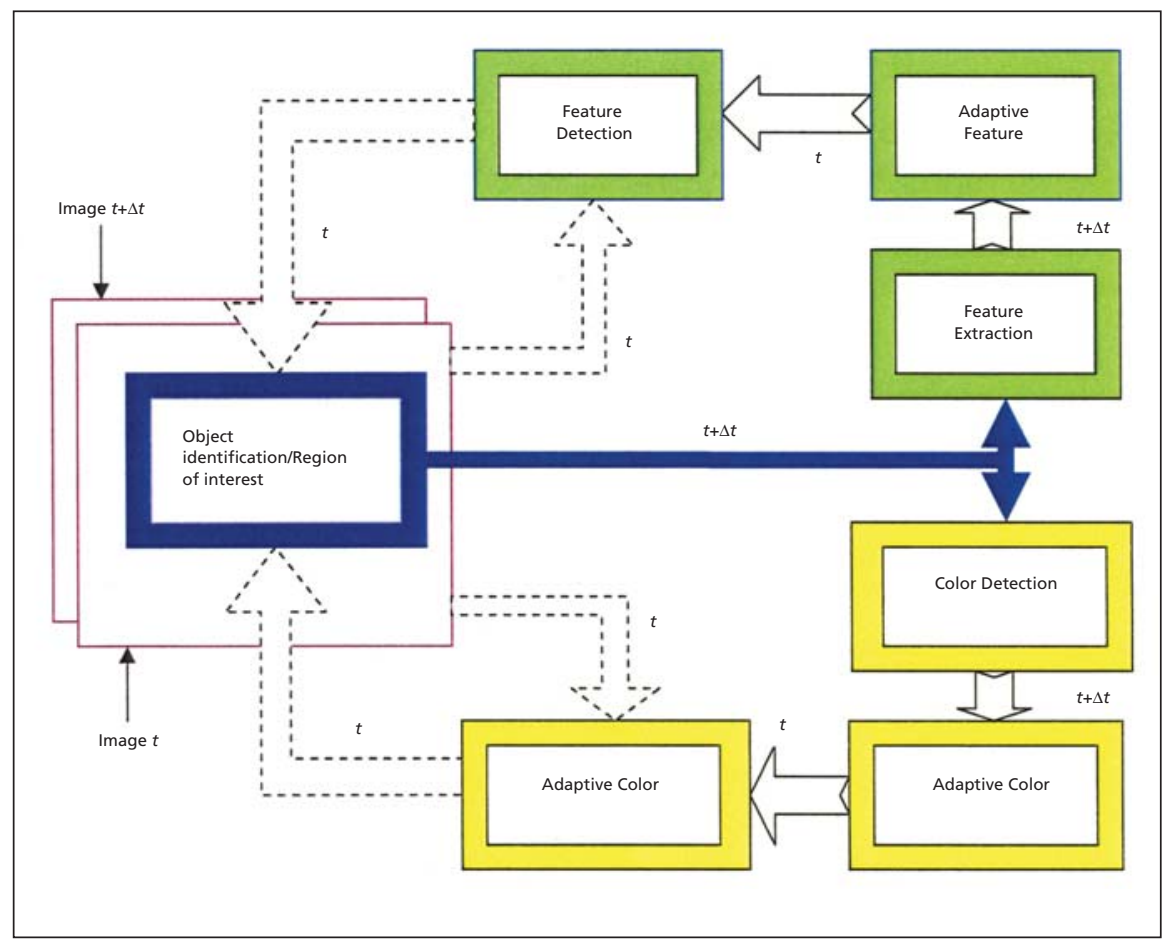

The Color and Shape Feature Feedback Adaptive architecture.

Laboratory. For more information, contact iaoffice@jpl.nasa.gov.

In accordance with Public Law 96-517, the contractor has elected to retain title to this invention. Inquiries concerning rights for its commercial use should be addressed to:

Innovative Technology Assets Management $J P L$
Mail Stop 202-233

4800 Oak Grove Drive

Pasadena, CA 91109-8099

E-mail: iaoffice@jpl.nasa.gov

Refer to NPO-47065, volume and number of this NASA Tech Briefs issue, and the page number.

\section{E Explanation Capabilities for Behavior-Based Robot Control}

\section{This mathematical framework can be applied to search and rescue or remote exploration robotic systems.}

\section{NASA's Jet Propulsion Laboratory, Pasadena, California}

A recent study that evaluated issues associated with remote interaction with an autonomous vehicle within the framework of grounding found that missing contextual information led to uncertainty in the interpretation of collected data, and so introduced errors into the command logic of the vehicle. As the vehicles became more autonomous through the activation of additional capabilities, more errors were made. This is an inefficient use of the platform, since the behavior of remotely located autonomous vehicles didn't coincide with the "mental models" of human operators.

One of the conclusions of the study was that there should be a way for the autonomous vehicles to describe what 